\title{
Determinantes sociais da saúde relacionados ao desmame precoce
}

\author{
Social determinants of health related to early weaning \\ Érica Oliveira Evangelista ${ }^{1}$, Lívia Keismanas de Ávila ${ }^{2}$
}

\section{Resumo}

Objetivo: Identificar na literatura científica os determinantes sociais da saúde que influenciam o desmame precoce. Método: Estudo bibliográfico, descritivo, de abordagem qualitativa e quantitativa, realizado nas bases de dados Literatura-americana e do Caribe em Ciências da Saúde, Bases de Dados de Enfermagem e na Scientific Eletronic Library Online. Foram incluídos no estudo artigos científicos publicados nos últimos 10 anos, em português (Brasil), que mencionaram fatores que determinam o desmame precoce. Os dados foram analisados utilizando a técnica de análise de conteúdo que consiste num processo de redução de texto em palavras e expressões significativas. Resultados: Após relacionarmos as expressões temáticas identificadas com a prevalência dos Determinantes Sociais da Saúde (DSS), identificamos que se sobressaiu os determinates relacionados aos serviços de saúde. Esses artigos referem que os serviços de saúde não adotam estratégias resolutivas para aumentar o tempo de duração do aleitamento materno exclusivo até os seis meses de vida da criança e que os profissionais possuem habilidades e conhecimento insuficientes para orientação adequada à gestante e puérpera sobre o aleitamento materno. Conclusão: O esquema das relações hierárquicas entre os diversos DSS nos auxilia na identificação de lacunas na formulação de políticas públicas e possibilita o planejamento de ações direcionadas as necessidades nesses níveis, por isso é fundamental que o profissional enfermeiro incorpore em sua prática a identificação e valorização dos DSS ampliando sua visão assistencial para além do biológico.

1. Graduada em Enfermagem pela Faculdade de Ciências Médicas da Santa Casa de São Paulo

2. Professora Adjunta da Faculdade de Ciências Médicas da Santa Casa de São Paulo. Curso de Graduação em Enfermagem

Trabalho realizado: Faculdade de Ciências Médicas da Santa Casa de São Paulo. Curso de Graduação em Enfermagem

Endereço para correspondência: Lívia Keismanas de Ávila. Faculdade de Ciências Médicas da Santa Casa de São Paulo. Rua Dr. Cesário Mota Jr, 61 - $9^{\circ}$ andar - Vila Buarque - 01221020 - São Paulo - SP - Brasil. Fone: (11) 3367-7799. E-mail: livia.avila@fcmsantacasasp.edu.br
Descritores: Aleitamento materno, Desmame, Determinantes sociais da saúde

\begin{abstract}
Objective: Identify in scientific literacture the social determinants of health that influenced the early weaning. Method: Descriptive bibliographic review, in a quantitative and qualitative approach, performed in the data basis Literatura-americana e do Caribe em Ciências da Saúde, Bases de Dados de Enfermagem and Scientific Eletronic Library Online. Scientific articles in portuguese (Brazil) of the last 10 years were included, which mentioned factors that determine the early weaning. Data was analyzed using the content analysis method, which consists in a tex reduction process into significative words and/or expressions. Results: thematic expressions were identified and listed with Social Determinants of Health (SDH). Then it was remarked the determinats related to heath services. The included articles indicate that the health services do not adopt resolutive strategies to enhance the exclusive breast feeding period until the age of six months, and the professionals lack enough knowledge to perform an adequate guidance do pregnant and puerperal about breast feeding. Conclusion: The scheme of hierarchic relations amongst several SDH may help in the identification of lacunae in public policy formulation and allows the planning os specific actions towards the needs. Thus, it is mandatory that the nurse professional assume the identification and improvement of SDH in practice, shifting the assitential beyond the biological approach.
\end{abstract}

Key words: Breast feeding, Weaning, Social determinants of health

\section{Introdução}

A recomendação da Organização Mundial de Saúde (OMS), é que a prática de amamentar exclusivamente seja até os seis primeiros meses de vida da criança, e após esta idade seja complementada com outros tipos de alimentos podendo ser estendida até os dois anos ou mais, uma vez que leite materno é completo ${ }^{(1)}$. 
Amamentar é um ato natural, são inúmeras as vantagens para mãe e para o bebê, umas das principais é a criação do vínculo afetivo entre o binômio mãe e filho $^{(1-2)}$.

A criança também será protegida de alergias, diarreias, infecções, ganhará peso mais rápido, além de ficar menos tempo internada caso haja a necessidade, o que implicara em menos gastos para a saúde pública. A criança que é amamenta apresenta no futuro menos risco de doenças do tipo, hipertensão, colesterol alto, diabetes, obesidade ${ }^{(2)}$.

Os benefícios da amamentação se estendem também à mãe que perderá peso rapidamente, o útero recuperará o seu tamanho normal após o parto diminuindo assim o risco de possíveis hemorragias e consequentes anemias, além de cada ano que a mãe amamenta o risco de câncer de mama invasivo é reduzido para $6 \%$.

Em matéria recente noticiada pela revista britânica The Lancet o Brasil é referência mundial em aleitamento materno. A revista analisou dados sobre aleitamento em 153 países destacando as políticas públicas brasileira como modelo, anunciando que a redução da mortalidade infantil no Brasil foi $20 \%$ maior que a média mundial, segundo Organização Mundial da Saúde $(\mathrm{OMS})^{(2)}$.

Apesar desse aumento paulatino nas taxas de amamentação, ainda existe um fator preocupante queé o chamado desmame precoce, pois manter amamentação exclusiva até os seis meses não é uma tarefa fácil. A lactação por si é considerada um fenômeno complexo, e pode sofrer influencias por diversos fatores; "dentre eles destacam se sociais, demográficos, culturais e psicológicos da mãe e da família, que impactam sua duração"(3).

Esses fatores, segundo definição da Organização Mundial de Saúde (OMS) são chamados de Determinantes Sociais da Saúde (DSS), pois se relacionam com as condições de vida e de trabalho da pessoa. Tais DSS não podem ser analisados isoladamente, eles influenciam não somente nas doenças e riscos à população, vai além, influenciam tanto o indivíduo quanto a coletividade que ele se insere ${ }^{(4-5)}$.

O modelo de Dahlgren e Whitehead é um dos modelos que esquematiza as relações de determinantes sociais e a situação de saúde. Ele é um modelo simples, fácil de ser compreendido por diversos públicos, que contém uma clara visualização gráfica dos determinantes sociais da saúde (Ilustração 1$)^{(5)}$.

Neste modelo, os determinantes sociais da saúde (DSS) são expostos em camadas segundo o seu nível de abrangência. Na camada mais próxima, considerada a base, estão os determinantes individuais que são idade, sexo, e fatores genéticos que influenciam sobre seu potencial e as condições de vida ${ }^{(5)}$. Na primeira camada acima, está o comportamento e os estilos de vida dos indivíduos, nestes, o comportamento não dependem somente do indivíduo, mas dos determinantes sociais, como informação e o acesso, propaganda, acesso e condições de alimentação saudável, lazer, etc ${ }^{(5)}$.

Na segunda camada está a questão das redes comunitárias e de apoio, ou seja, as comunidades e suas redes de relações, trata-se de políticas que busquem estabelecer redes de apoio e fortalecer a organização e participação das pessoas e das comunidades $^{5}$. Na terceira camada estão os fatores de vida relacionados a condição de vida e de trabalho, ou seja, a produção agrícola e de alimentos, educação, ambiente, desemprego, água e esgoto, serviços sociais de saúde, habitação. No último nível da figura estão os considerados macrodeterminates, relacionados as condições socioeconômica, culturais e ambientais gerais, possuem também o poder de grande influência sobre as demais ${ }^{(5)}$

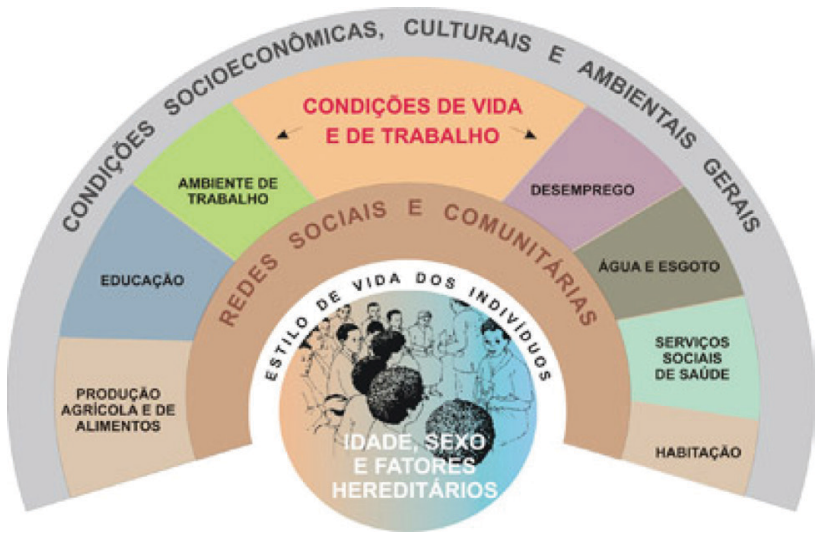

Ilustração 1 - Esquema de visualização de Dahlgren e Whitehead das relações hierárquicas entre os diversos determinantes da saúde. (Fundação Oswaldo Cruz, 2014)(4)

Diante disso a finalidade desse estudo é identificar os Determinantes Sociais da Saúde envolvidos no desmame precoce, com o intuito de divulgar estratégias da prática do profissional enfermeiro na assistência à saúde da mulher, bem como contribuir com o desenvolvimento de programas e políticas públicas de atenção à saúde da mulher aumentando assim as taxas e índices de amamentação exclusiva no país.

\section{Método}

Estudo bibliográfico, descritivo, de abordagem, qualitativo e quantitativo ${ }^{(6)}$. O estudo foi realizado nas bases de dados Literatura-americana e do Caribe em Ciências da Saúde (LILACS), e Bases de Dados De Enfermagem (BDENF), e na Scientific Eletronic Library Online (SciELO).

Foram incluídos no estudo artigos científicos 
publicados nos últimos 10 anos, em português, que mencionaram fatores que determinam o desmame precoce

Foi realizada a partir da relação entre as palavras chave: aleitamento materno, amamentação e desmame precoce. Após identificação das publicações, as mesmas foram submetidas a análise do título e resumo para averiguação da pertinência com o objeto de pesquisa, em seguida os artigos selecionados foram lidos na íntegra.

Os dados foram analisados utilizando a técnica de análise de conteúdo que segundo Minayo(2010) ${ }^{(7)}$, consiste num processo de redução de texto em palavras e expressões significativas.

\section{Resultados}

Ao relacionarmos as palavras chave na busca avançada da Biblioteca Virtual em Saúde -BVS - (Lilacs e BDENF) e no SciELO, foram identificados 70 publicações. Após a aplicação dos critérios de inclusão estabelecidos foram selecionados 20 artigos científicos.

\section{Discussão}

Ao relacionarmos as expressões-temática das publicações identificadas com a prevalência dos Determinastes Sociais da Saúde (DSS) identificamos que se sobressai Serviços de Saúde conforme mostra a figura 1. Para organização da discussão, optamos por apresentar a prevalência dos DSS a partir do modelo hierárquico de Dahlgren e Whitehead.

Na figura do modelo de Dahlgren e Whitehead com as relações hierárquicas dos DSS, na base do modelo envolve idade, sexo, fatores hereditários, fatores psicológicos o que segundo descrição do modelo exerce influência sobre o potencial e as condições de saúde dos indivíduos, também denominado características individuais ${ }^{(8)}$

Com relação a idade, as mulheres mais jovens estão mais susceptíveis a desmamarem precocemente devido ao despreparo, falta de experiência. Estudos apontam que crianças nascidas de mulheres com mais idade, tiveram mais chance de serem amamentadas até os seis meses ${ }^{(9)}$.

No nível seguinte, considera-se as questões relacionadas ao comportamento que podem ser influenciadas por meio do acesso a informação. Estas características são definidas por estilo de vida dos indivíduos dentro da classificação dos DSS, encontradas durante o estudo nas mulheres lactantes, e que são fatores para o desmame precoce, são referentes aos problemas nas mamas como trauma mamilar, ingurgitamento, dor ao amamentar, fissuras, bebê com dificuldade de pega.

Autores apontam que estas causas estão relacionadas com a falta de orientação adequada pelos profissionais e serviços de saúde que as acompanha' associada a falta de preparo dos mesmos para orientar as nutrizes sobre os procedimentos a serem adotados principalmente sobre os relacionados a estimulação da produção e fisiologia da lactação $0^{(9-10)}$.

O parto cesária, já considerado como epidemia no país, ultrapassa a proporção de $10 \%$ a $15 \%$ recomendada pela OMS e está incluso dentro do modelo como Estilo de Vida, sendo realizado, na maioria dos casos, de forma eletiva sem a real indicação, necessidade e fortemente ligado ao desmame. Este tipo de parto

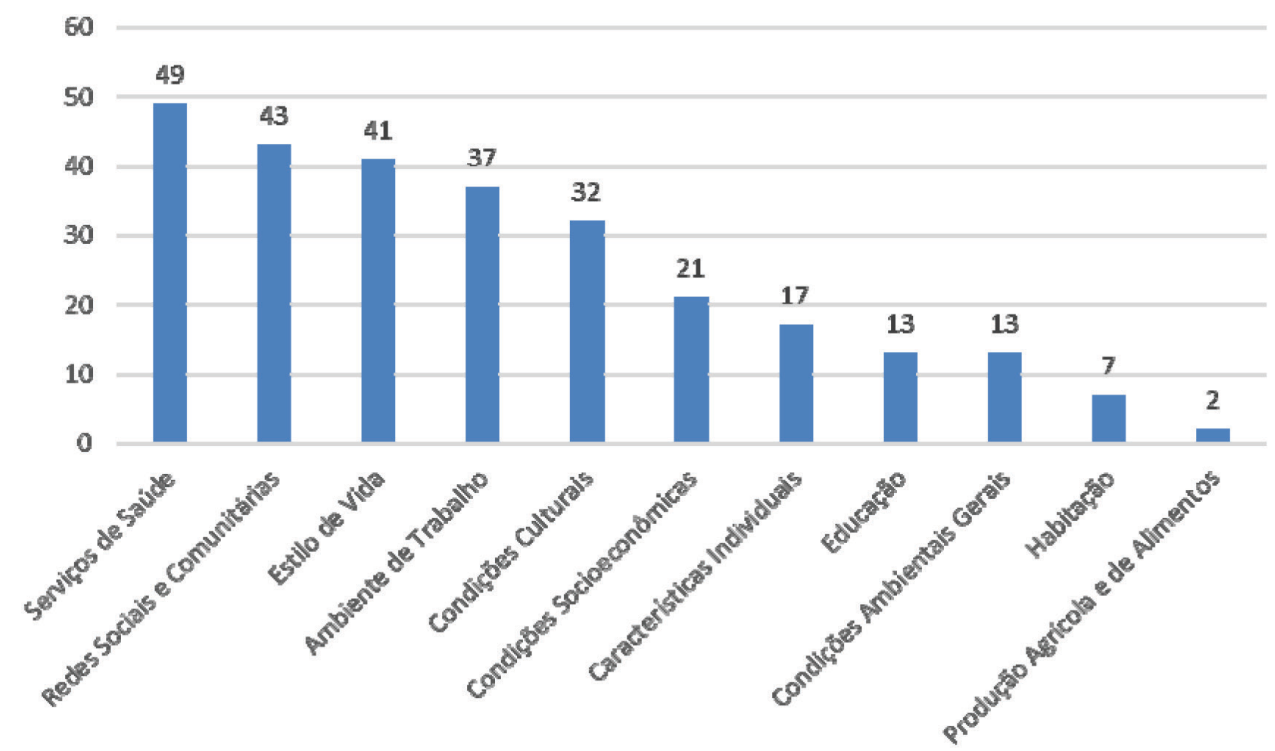

Figura 1 - Distribuição dos artigos identificados no estudo referente à amamentação e desmame precoce segundo Prevalência dos Determinantes Sociais de Saúde. Brasil. abril e maio 2016. 
Evangelista EO, Ávila LK. Determinates sociais da saúde relacionados ao desmame precoce. Arq Med Hosp Fac Cienc Med Santa Casa São Paulo. 2018;63(1):40-4.

dificulta a ida para o alojamento conjunto e o início precoce da amamentação ${ }^{(11)}$.

Na segunda camada referente às redes sociais e comunitárias que corresponde à comunidade e suas redes de relações onde os laços de coesão social e as relações de solidariedade e confiança entre pessoas e grupos são fundamentais para a promoção e proteção da saúde individual e coletiva. Neste foram encontradas expressões referentes as seguintes condições, o apoio da mãe, da avó, do companheiro e até mesmo dos serviços de saúde, como das instituições que possuem certificado de Iniciativa Hospital Amigo da Criança (IHAC) $9^{(8,12)}$.

A próxima camada envolve os fatores condicionantes da saúde relacionados a condições de vida e de trabalho, disponibilidade de alimentos e acesso a ambientes e serviços essenciais, como saúde e educação, mais ambiente de trabalho, desemprego, água e esgoto e habitação. Com relação à habitação os estudos apontam que Região Nordeste possui a taxa mais baixa de $\mathrm{AME}^{(13)}$. As mulheres que residem em zona urbana à duração do AME é menor em comparação das que vivem na zona rural ${ }^{(14-16)}$.

Com relação aos serviços de saúde, DSS predominantemente encontrado no estudo como fator responsável pelo desmame precoce, foi identificado que serviços de saúde não adotam estratégias resolutivas para aumentar duração do AME até os seis meses de vida da criança, profissionais possuem conhecimento e habilidades insuficientes sobre amamentação para orientar adequadamente ${ }^{(14)}$.

Quanto ao ambiente de trabalho, entre os fatores que dificultam o AME, destaca-se trabalho noturno, extensa jornada de trabalho, condições insalubres para a ordenha do leite no local de trabalho e falta de informação e interesse da empresa quanto às políticas de $\mathrm{AM}^{(15)}$. Sobre a educação, a baixa escolaridade é um fator determinante para a prática da amamentação e a continuação, mulheres com mais anos de estudos amamentam seus filhos por mais tempo do que as que têm poucos anos ${ }^{(15)}$.

A disponibilidade de alimentos para a nutriz , pouco citada nos artigos, e de suma importância, uma vez que influencia no AME devido a exigência nutricional da mulher que amamenta, sabemos que nem sempre o acesso aos alimentos é possível devido às condições financeiras da parturiente ${ }^{(15)}$.

No último nível estão os macrodeterminantes que são as condições socioeconômicas, culturais e ambientais gerais. A situação socioeconômica está relacionada com ao baixo poder aquisitivo que a família ou a parturiente possui, em geral, com renda mensal inferior a um salário mínimo. Além disso, destacam-se as mulheres solteiras, que consequentemente são as mantenedoras da família, e por isso tem risco de desmame precoce por medo de perder o emprego ${ }^{(15)}$. Mitos e crenças sobre o valor nutricional do leite materno, como achar que é fraco, não alimentar o bebê e o uso de chupetas e mamadeiras entram nos fatores das condições culturais ${ }^{(13,16)}$. Como exemplo de condições ambientais gerais foram encontradas diversas situações que podem influenciar AME como famílias numerosas, a introdução da tecnologia, o fato de a mulher hoje ter um cotidiano mais ansioso e tenso ${ }^{(12-13,17)}$.

\section{Considerações Finais}

Conclui-se que o esquema de visualização de Dahlgren e Whitehead das relações hierárquicas entre os diversos DSS, possibilita definir políticas para os pontos que necessite de intervenções dentro das camadas.

No primeiro nível do modelo dos DSS, é necessário criar ações que irão atuar sobre o comportamento das puérperas, pois neste nível estão envolvidos fatores comportamentais e estilo de vida. No segundo nível, redes sociais e comunitárias, é importante que se inclua políticas públicas intersetoriais que estabeleçam redes de apoio, participação familiar e das organizações de saúde, para o "empoderamento" da mulher que acabou de dar à luz e precisa sentir-se segura quanto as questões da amamentação.

O terceiro nível trata-se de condições de vida e trabalho. Neste âmbito é necessário atuar com políticas em setores que são distintos, dentre eles o setor saúde, que assiste as gestantes e puérperas, como hospitais e unidades básicas de saúde; as empresas (trabalho e emprego) que mantém vínculos empregatícios com essas nutrizes; e no setor da educação, para que se possibilite mais anos de estudos com qualidade, reduzindo assim a possibilidade de desmame precoce. Necessita então de mecanismos que possibilite a ação de todos juntos, de forma integrada, em redes, para viabilizar uma adequada assistência à mulher gestante/puérpera, recém-nascido e família, com todos os seus direitos referente à amamentação esclarecidos e garantidos.

Neste nível, ainda se verifica a necessidade de mudanças imediatas nos serviços de saúde, e isto envolve desde a gestão institucional até as competências e habilidades dos profissionais, bem como suas atribuições, pois estes se constituem peças chave para modificar o atual cenário do desmame precoce.

No quarto nível do modelo o indicado é a criação de políticas públicas voltadas a promoção da saúde pois estas evolvem todos os denominados macrodeterminantes. Estas políticas devem considerar a dimensão da interculturalidade, considerando os valores e crenças potenciais na manutenção do aleitamento 
materno e transformando os que não contribuem positivamente.

Além disso, deve-se reduzir iniquidades sociais contribuindo para condições socioeconômicas dignas, por meio de políticas públicas que estabeleçam oportunidade e distribuição de renda com equidade.

Também incluído no nível que envolve os demais determinantes sociais da saúde, têm-se as condições ambientais gerais, que se constitui por fatores relacionados ao o que está acontecendo no país e no mundo, que muitas vezes influencia e determina comportamento, acesso, disponibilidade e tipos de serviços, e que envolve todos os contextos que esta mulher está inserida, em uma sociedade globalizada.

Assim, é fundamental o profissional enfermeiro incorporar em sua prática profissional a identificação e valorização dos determinantes sociais da saúde no intuito de ampliar a sua visão assistencial para além do biológico.

\section{Referências}

1. Barros SMO. Enfermagem no ciclo gravídico-puerperal. Barueri, SP: Manole; 2006. 259p.

2. Frasão G. Brasil segue sendo referência mundial em aleitamento, afirma The Lancet. 29/01/2016 [online]. Disponível em: http:/ / portalsaude.saude.gov.br/index.php/cidadao/principal/ agencia-saude/21939-brasil-segue-sendo-referencia-mundial-em-aleitamento-afirma-the-lancet (13 fev 2016)

3. Almeida ISA, Pugliesi Y, Rosado LEP. Estratégias de promoção e manutenção do aleitamento materno baseadas em evidência: revisão sistemática. Femina. 2015; 43(3):98-102.

4. Fundação Osvaldo Cruz (FIOCRUZ). Determinantes Sociais. Renast Online. [online]. Rio de Janeiro: FIOCRUZ; 2014. Disponível em: http:/ / pensesus.fiocruz.br/determinantes-sociais. (22 fev 2016)

5. Comissão Nacional sobre Determinantes Sociais da Saúde. Análise da situação de saúde. Comissão Nacional sobre Determinantes Sociais da Saúde. As causas sociais das iniquidades em saúde no Brasil. Rio de Janeiro: Fiocruz; 2008. p.17-21.
6. Gil AC. Como elaborar projetos de pesquisa. $4^{\mathrm{a}}$ ed. São Paulo: Atlas; 2002. 184p.

7. Minayo MCS. O desafio do conhecimento pesquisa qualitativa em saúde. São Paulo: Hucitec; 2010. 407p.

8. Buss PM, Pellegrini Filho A. A saúde e seus determinantes sociais. Physis (Rio J.). 2007; 17(1):77-93.

9. Stephan AMS, Cavada MN, Vilela CZ. Prevalência de aleitamento materno exclusivo até a idade de seis meses e características maternas associadas, em área de abrangência de unidade de Saúde da Família no Município de Pelotas, Estado do Rio Grande do Sul, Brasil, 2010. Epidemiol Serv Saúde. 2012; 21(3):431-8.

10. Giuliani NR, Oliveira J, Bosco VL. O início do desmame precoce: motivos das mães assistidas por serviços de puericultura de Florianópolis/SC para esta prática. Pesqui Bras Odontopediatria Clin Integr. 2012; 12(1):53-8.

11. Wil TK, Arndt JG, Torres GG, Andrade JR, Pereira TSS, Molina MCB. Fatores de proteção para a amamentação na primeira hora de vida. Rev Bras Promoç Saúde (Impr.). 2013; 26(2):274-80.

12. Araújo OD, Cunha AL, Lustosa LR, Nery IS, Mendonça RCM, Campelo SMA. Aleitamento materno: fatores que levam ao desmame precoce. Rev Bras Enferm. 2008; 61(4):488-92.

13. Freitas LJQ, Melo NCCC, Valente MMQP, Moura ERF, Américo CF, Sousa CSP. Amamentação ineficaz entre nutrizes atendidas em unidades básicas de saúde. Rev Enferm UERJ. 2014; 22(1):103-10.

14. Demétrio F, Pinto EJ, Assis AMO. Fatores associados à interrupção precoce do aleitamento materno: um estudo de coorte de nascimento em dois municípios do Recôncavo da Bahia, Brasil. Cad Saúde Pública. 2012; 28(4):641-54

15. Silva CA, Davim RMB. Mulher trabalhadora e fatores que interferem na amamentação: revisão integrativa. Rev Rene. 2012; 13:1208-17.

16. Brasileiro AA, Ambrosano GMB, Marba STM, Passon RF. A amamentação entre filhos de mulheres trabalhadoras. Rev Saúde Pública. 2012; 46(4):642-8.

17. Fonseca-Machado MO, Haas VJ, Stefanello J, Nakano AMS, Sponholz FG. Aleitamento materno: conhecimento e prática. Rev Esc Enferm USP. 2012; 46(4):809-15.

Trabalho recebido: $21 / 11 / 2016$

Trabalho aprovado: 25/04/2018 